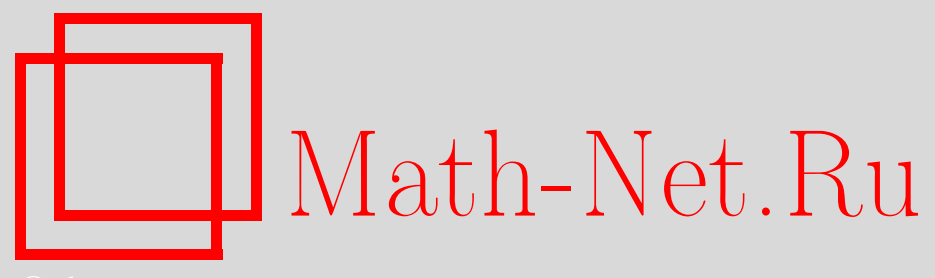

В. А. Копытцев, О числе решений системы случайных линейных уравнений в множестве векторов специального вида, Дискрет. матем., 2006, том 18, выпуск 1, 40-62

DOI: https://doi.org/10.4213/dm31

Использование Общероссийского математического портала Math-Net.Ru подразумевает, что вы прочитали и согласны с пользовательским соглашением http://www . mathnet.ru/rus/agreement

Параметры загрузки:

IP: 54.80 .73 .141

26 апреля 2023 г., 12:30:59

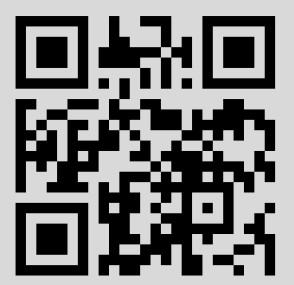


УдК 519.2

\title{
О числе решений системы случайных линейных уравнений в множестве векторов специального вида
}

\author{
(c) 2006 г. $\quad$ В. А. Копытцев
}

Исследуется распределение числа решений системы случайных линейных уравнений над $G F(q)$ в множестве векторов, обладающих заданным числом ненулевых координат, а также в некоторых подмножествах этого множества. Получены достаточные условия сходимости распределений к закону Пуассона, к некоторым другим предельным распределениям, связанным с этим законом, и условия сходимости к стандартному нормальному закону. В работе обобщены результаты, доказанные автором ранее для случая $q=2$.

\section{1. Введение и формулировки результатов}

Пусть компоненты вектора $x=\left(x_{1}, \ldots, x_{n}\right)$ принимают значения в конечном поле $G F(q)$, $q \geqslant 2$. Положим

$$
X_{r}=\left\{x: \sum_{i=1}^{n} I\left(x_{i}\right)=r\right\}
$$

где $r \in\{0,1, \ldots, n\}$ и

$$
I\left(x_{i}\right)= \begin{cases}1, & x_{i} \in G F(q) \backslash\{0\}, \\ 0, & x_{i}=0 .\end{cases}
$$

Очевидно, что число элементов множества (1)

$$
\left|X_{r}\right|=\left(\begin{array}{l}
n \\
r
\end{array}\right)(q-1)^{r}
$$

Пусть $X_{r}^{\prime}$ - произвольное подмножество множества $X_{r}$ с числом элементов

$$
\left|X_{r}^{\prime}\right| \geqslant\left(\begin{array}{l}
n \\
r
\end{array}\right) \text {. }
$$

В частности, можно полагать, что

$$
X_{r}^{\prime}=\left\{x: \sum_{i=1}^{n} I_{B}\left(x_{i}\right)=r, \sum_{i=1}^{n} I_{0}\left(x_{i}\right)=n-r\right\},
$$


где $r \in\{0,1, \ldots, n\}$ и

$$
\begin{gathered}
I_{B}\left(x_{i}\right)= \begin{cases}1, & x_{i} \in B, \\
0, & x_{i} \notin B,\end{cases} \\
I_{0}\left(x_{i}\right)= \begin{cases}1, & x_{i}=0, \\
0, & x_{i} \neq 0,\end{cases}
\end{gathered}
$$

и $B$ - некоторое подмножество множества ненулевых элементов поля $G F(q)$. В этом случае

$$
\left|X_{r}^{\prime}\right|=\left(\begin{array}{l}
n \\
r
\end{array}\right)|B|^{r}, \quad 1 \leqslant|B| \leqslant q-1 .
$$

Рассмотрим систему случайных линейных уравнений над полем $G F(q)$

$$
\varepsilon_{t, 1} x_{1}+\ldots+\varepsilon_{t, n} x_{n}=a_{t}, \quad t=1, \ldots, T,
$$

с ограничением

$$
x=\left(x_{1}, \ldots, x_{n}\right) \in X_{r}^{\prime},
$$

где $r-$ заданное натуральное число, $1 \leqslant r \leqslant n-1$. Коэффициенты $\varepsilon_{t, i}, i=1, \ldots, n$, $t=1, \ldots, T$, предполагаются независимыми в совокупности случайными величинами,

$$
\begin{aligned}
\mathbf{P}\left\{\varepsilon_{t, i}=\nu\right\} & =\frac{1}{q}\left(1+\Delta_{t, i}^{(v)}\right), \\
\sum_{\nu \in G F(q)} \Delta_{t, i}^{(v)} & =0 .
\end{aligned}
$$

Следуя работе [2], назовем систему уравнений (4) (с ограничением (5)) случайной системой с независимыми частями, если вектор правых частей $a=\left(a_{1}, \ldots, a_{T}\right)$ не зависит от коэффициентов $\varepsilon_{t, i}, i=1, \ldots, n, t=1, \ldots, T$. Распределение

$$
\mathbf{P}_{T}\{a=\alpha\}, \quad \alpha \in G F(q)^{T},
$$

вектора $a$ может быть произвольным, в том числе, сосредоточенным на некотором одном значении $\alpha \in G F(q)^{T}$.

Систему уравнений (4) назовем случайной заведомо совместной системой, если

$$
a_{t}=\varepsilon_{t, 1} x_{1}^{0} \oplus \ldots \oplus \varepsilon_{t, n} x_{n}^{0}, \quad t=1, \ldots, T,
$$

при некотором заданном векторе $x^{0}=\left(x_{1}^{0}, \ldots, x_{n}^{0}\right) \in X_{r}^{\prime}$. Вектор $x^{0}$ назовем истинным решением.

Обозначим $\xi$ и $\eta$, соответственно, число решений случайной системы с независимыми частями и число решений случайной заведомо совместной системы.

Отметим, что число $\xi$ является функцией случайной матрицы коэффициентов $E=\left\|\varepsilon_{t, i}\right\|$ и (в общем случае) случайного вектора $a=\left(a_{1}, \ldots, a_{T}\right)$, а число $\eta$ является функцией случайной матрицы $E$. Исходя из этого, будем полагать, что

$$
\begin{aligned}
& \xi=f(E, a \mid r), \\
& \eta=g\left(E \mid x^{0}, r\right),
\end{aligned}
$$


где $r$ и $x^{0}$ - заданные параметры, $f$ и $g-$ заданные функции, $E$ и $a$, соответственно, случайная матрица и случайный вектор.

Пусть

$$
\begin{aligned}
& \Lambda=\left|X_{r}^{\prime}\right| q^{-T}, \\
& \Delta=\max _{t, i, v}\left|\Delta_{t, i}^{(v)}\right| .
\end{aligned}
$$

Для обозначения предельных соотношений вида

$$
\mathbf{P}\{\xi=k\} \rightarrow \frac{\lambda^{k} e^{-\lambda}}{k !}, \quad k=0,1,2, \ldots,
$$

будем использовать запись $\xi \rightarrow \Pi(\lambda)$ и запись $\xi \rightarrow \mathcal{N}(0,1)$ для обозначения сходимости функщии распределения случайной величины $\xi$ к функщии стандартного нормального распределения.

Теорема 1. Пусть $n, T \rightarrow \infty, r / n \rightarrow 0, \Delta \rightarrow 0, T \Delta^{r} \rightarrow 0$,

$$
\Lambda \rightarrow \lambda<\infty \text {. }
$$

Тогда

$$
\xi \rightarrow \Pi(\lambda)
$$

равномерно относительно $a \in G F(q)^{T}, a \neq 0$.

Пусть $a=0, X_{r}^{\prime}=X_{r}$ и выполняются условия первой части теоремы. Тогда

$$
\frac{1}{q-1} \xi \rightarrow \Pi(\lambda /(q-1))
$$

Пусть в подмножестве $X_{r}^{\prime}$ не содержится пар коллинеарных векторов и выполняются условия первой части теоремы. Тогда выполняется соотночение (12) равномерно относительно а $\in$ GF $(q)^{T}$.

Замечание 1. Условию третъей части теоремы 1 удовлетворяет, к примеру, подмножество $X_{r}^{\prime}$, содержащее по одному (произвольному) представителю из каждого класса коллинеарных векторов, на которые разбивается множество $X_{r}$. При этом

$$
\left|X_{r}^{\prime}\right|=\left(\begin{array}{l}
n \\
r
\end{array}\right)(q-1)^{r-1}
$$

Отметим, что в случае $q=2$ множество $X_{r}^{\prime}$ в силу требования (2) совпадает с $X_{r}$ и не содержит пар коллинеарных векторов, так как в данном случае такие пары отсутствуют вообще.

Положим

$$
\Pi(k, \lambda)=\frac{\lambda^{k} e^{-\lambda}}{k !}, \quad k=0,1, \ldots
$$

Следствие 1. Пусть $X_{r}^{\prime}=X_{r}$ и выполняются условия первой части теоремы 1. Тогда

$$
\mathbf{P}\{\xi=k\}=\mathbf{P}\{a \neq 0\} \Pi(k, \lambda)+\mathbf{P}\{a=0\} \Pi(k /(q-1), \lambda /(q-1))+o(1),
$$

если q-1 делит $k \in\{1,2, \ldots\}, u$

$$
\mathbf{P}\{\xi=k\}=\mathbf{P}\{a \neq 0\} \Pi(k, \lambda)+o(1),
$$

если $q-1$ не делит $k \in\{1,2, \ldots\}$. 
Следствие 2. Пусть вектор а распределен равномерно, то есть

$$
\mathbf{P}\{a=\alpha\}=q^{-T}, \quad \alpha \in G F(q)^{T},
$$

и выполняются условия первой части теоремы 1. Тогда

$$
\xi \rightarrow \Pi(\lambda) .
$$

Теорема 2. Пусть $X_{r}^{\prime}$ определяется формулой (3), $|B|=1$, вектор а распределен равномерно, $n, T \rightarrow \infty,(n-r) / n \rightarrow 0, \Delta \rightarrow 0, T \Delta^{n-r} \rightarrow 0, \Lambda \rightarrow \lambda<\infty$. Тогда имеет место соотношение (14).

Теорема 3. Пусть выполняется одно из условий

(a) $\Delta=0$, или

(б) $X_{r}^{\prime}$ определяется по формуле (3), $|B|=1, \Delta_{t, i}^{(v)}=\Delta_{t}^{(v)}, i=1, \ldots, n$.

Тогда распределение числа решений $\eta=g\left(E \mid x^{0}, r\right)$ инвариантно относительно $x^{0} \in X_{r}^{\prime}$.

Теорема 4. Пусть выполняется одно из условий

(в) $\Delta=0, n, T \rightarrow \infty, r / n \rightarrow 0, \Lambda \rightarrow \lambda<\infty$,

(г) $X_{r}^{\prime}$ определяется формулой (3), $|B|=1, \Delta_{t, i}^{(v)}=\Delta_{t}^{(v)}, i=1, \ldots, n$, и при этом выполняются условия первой части теоремы 1.

Tогда

$$
\eta-1 \rightarrow \Pi(\lambda)
$$

Теорема 5. Пусть выполняется условие (б) теоремы $3, n, T \rightarrow \infty,(n-r) / n \rightarrow 0, \Delta \rightarrow 0$, $T \Delta^{n-r} \rightarrow 0, \Lambda \rightarrow \lambda<\infty$.

Тогда имеет место соотночение (15).

Теорема 6. Пусть $n, T \rightarrow \infty, r / n \rightarrow 0, \Delta \rightarrow 0, T \Delta^{r} \rightarrow 0$,

$$
\begin{gathered}
\Lambda=\left|X_{r}^{\prime}\right| q^{-T} \rightarrow \infty, \\
\ln \Lambda / r \ln (n / r) \rightarrow 0
\end{gathered}
$$

и в случае $\Delta>0$

$$
\ln \Lambda / \ln \left(T \Delta^{r}\right) \rightarrow 0
$$

Тогда

$$
(\xi-\Lambda) \Lambda^{-1 / 2} \rightarrow \mathcal{N}(0,1)
$$

равномерно по $а \in G F(q)^{T}, a \neq 0$.

Пусть $a=0, X_{r}^{\prime}=X_{r}$ и выполняются условия первой части теоремы. Тогда

$$
(\xi-\Lambda)(\Lambda(q-1))^{-1 / 2} \rightarrow \mathcal{N}(0,1) .
$$

Пусть подмножество $X_{r}^{\prime}$ не содержит пар коллинеарных векторов и выполняются условия первой части теоремы. Тогда выполняется соотношение (19) равномерно по $a \in G F(q)^{T}$. 
Теорема 7. Пусть $X_{r}^{\prime}$ определяется формулой (3), $|B|=1$, вектор а распределен равномерно и выполняются условия, полученные из условий первой части теоремы 6 заменой параметра $r$ параметром $n-r$. Тогда

$$
(\xi-\Lambda) \Lambda^{-1 / 2} \rightarrow \mathcal{N}(0,1) .
$$

Теорема 8. Пусть выполняется одно из условий

(д) $\Delta=0, n, T \rightarrow \infty, r / n \rightarrow 0$, и выполняются условия (16), (17),

(е) $X_{r}^{\prime}$ определяется формулой (3), $|B|=1, \Delta_{t, i}^{(v)}=\Delta_{t}^{(v)}, i=1, \ldots, n, u$ выполняются условия первой части теоремы 6.

Тогда

$$
(\eta-\Lambda) \Lambda^{-1 / 2} \rightarrow \mathcal{N}(0,1)
$$

Теорема 9. Пусть $X_{r}^{\prime}$ определяется формулой (3), $|B|=1, \Delta_{t, i}^{(v)}=\Delta_{t}^{(v)}, i=1, \ldots, n, u$ выполняются условия, полученные из условий первой части теоремы 6 заменой параметра $r$ параметром $n$ - $r$. Тогда имеет место соотношение (22).

Замечание 2. Наряду с ограничением (5) для решения системы уравнений (4) можно рассмотреть ограничения

$$
\begin{array}{ll}
x \in \bigcup_{k=1}^{r} X_{k}^{\prime}, & 1 \leqslant r \leqslant n-1, \\
x \in \bigcup_{k=r}^{r} X_{k}^{\prime}, & 1 \leqslant r \leqslant n-1,
\end{array}
$$

полагая при этом, что множества $X_{k}^{\prime}$ определяются формулой (3).

Обозначим $\xi^{(1)}$ и $\xi^{(2)}$ числа решений случайной системы (4) с независимыми частями, удовлетворяющих ограничениям (23) и (24), соответственно.

Обозначим $\eta^{(1)}$ и $\eta^{(2)}$ числа решений случайной заведомо совместной системы (4), удовлетворяющих ограничениям (23) и (24). При этом полагаем, что как в случае (23), так и в случае (24) истинным решением является $x^{0} \in X_{r}^{\prime}$.

Покажем, что для введенных величин справедливы утверждения, аналогичные теоремам 1-9 и отличающиеся только некоторыми дополнительными условиями.

Действительно, пусть выполняются условия первой части теоремы 1 . Случай $r=1$ тривиален, так как влечет равенство $\xi^{(1)}=\xi$. При $r \geqslant 2$ и условии, что

$$
T \Delta=O(1),
$$

математическое ожидание

$$
\begin{aligned}
\mathbf{E}\left(\xi^{(1)}-\xi\right) & \leqslant((1+\Delta) / q)^{T} \sum_{k=1}^{r-1}\left|X_{k}^{\prime}\right|-((1+\Delta) / q)^{T} \sum_{k=1}^{r-1}\left(\begin{array}{l}
n \\
k
\end{array}\right)|B|^{k} \\
& \leqslant(1+\Delta)^{T} \Lambda \sum_{l \geqslant 1}(r /(n-r))^{l} \rightarrow 0 .
\end{aligned}
$$


Поэтому согласно (12)

$$
\xi^{(1)} \rightarrow \Pi(\lambda)
$$

равномерно по $a, a \neq 0$.

В случае $a=0, X_{r}^{\prime}=X_{r}$ из (25) и (13) следует, что

$$
(1 /(q-1)) \xi^{(1)} \rightarrow \Pi(\lambda /(q-1)) .
$$

Если $X_{r}^{\prime}$ не содержит коллинеарных векторов, то из (18) и третьей части теоремы 1 следует соотношение (26), выполняемое равномерно по всем значениям $\alpha \in G F(q)^{T}$ вектора $a$.

По аналогии с (7) положим

$$
\xi^{(i)}=f^{(i)}(E, a \mid r), \quad i=1,2
$$

Утверждение для числа $\xi^{(2)}$, соответствующее теореме 2, вытекает из следующей леммы.

Лемма 1. Пусть подмножества $X_{k}^{\prime}$ в ограничениях (23), (24) определяются формулой (3), где $|B|=1$, и пусть вектор правых частей а распределен равномерно, тогда

$$
\mathbf{P}\left\{f^{(2)}(E, a \mid r)=l\right\}=\mathbf{P}\left\{f^{(1)}(E, a \mid n-r)=l\right\}, \quad l=0,1,2, \ldots
$$

Эта лемма доказывается так же, как лемма 12, используемая ниже при доказательстве теоремы 2.

Рассмотрим распределения чисел

$$
\eta^{(i)}=g^{(i)}\left(E \mid x^{0}, r\right), \quad i=1,2 .
$$

Сначала отметим, что справедливы утверждения, которые получаются из теоремы 3 заменой числа $\eta=g\left(E \mid x^{0}, r\right)$ на любое из чисел (29). Доказательство этих утверждений дословно повторяет доказательство теоремы 3.

Покажем теперь, как получается утверждение для числа $\eta^{(1)}$, соответствующее теореме 4. Случай $r=1$ тривиален, так как влечет равенство $\eta^{(1)}=\eta$.

Пусть

$$
x^{0} \in X_{r}^{\prime}, \quad r \geqslant 2, \quad x^{1} \in \bigcup_{k=1}^{r-1} X_{k}^{\prime}
$$

Вектор $x^{1}$ удовлетворяет заведомо совместной системе (4) с ограничением (23), если и только если скалярные произведения

$$
\left(\varepsilon_{t}, x^{0}-x^{1}\right)=0, \quad t=1, \ldots, T,
$$

где

$$
\begin{aligned}
\varepsilon_{t} & =\left(\varepsilon_{t, 1}, \ldots, \varepsilon_{t, n}\right) \\
x^{0}-x^{1} & =\left(x_{1}^{0}-x_{1}^{1}, \ldots, x_{n}^{0}-x_{n}^{1}\right) .
\end{aligned}
$$

При любом $x^{0} \in X_{r}^{\prime}$

$$
\mathbf{P}\left\{\left(\varepsilon_{t}, x^{0}-x^{1}\right)=0, t=1, \ldots, T\right\} \leqslant q^{-T}(1+\Delta)^{T} .
$$


Используя последнее неравенство, получаем, что если

$$
T \Delta=O(1), \quad r=o(n), \quad \Lambda \rightarrow \lambda, \quad \lambda<\infty,
$$

то выполняются соотношения, аналогичные соотношениям (25):

$$
\mathbf{E}\left(\eta^{(1)}-\eta\right) \leqslant(1+\Delta)^{T} \Lambda \sum_{l \geqslant 1}(r /(n-r))^{l} \rightarrow 0 .
$$

Пусть выполняется условие (г) теоремы 4 и

$$
T \Delta=O(1) .
$$

Тогда из (30) и (15) следует, что

$$
\eta^{(1)}-1 \rightarrow \Pi(\lambda)
$$

Пусть множество $X_{r}^{\prime}$ определяется формулой (3), $|B|=1, B=\{\beta\}$. Как нетрудно заметить, в этом случае

$$
g^{(2)}\left(E \mid x^{0}, r\right)=g^{(1)}\left(E \mid x^{1}, n-r\right),
$$

где

$$
x^{1}=\left(\beta-x_{1}^{0}, \ldots, \beta-x_{n}^{0}\right)
$$

Из (31), (32) вытекает утверждение для числа $\eta^{(2)}$, соответствующее теореме 5.

Аналогично получаются утверждения, соответствующие теоремам 6-9. При этом используются соотношения (25), (30), имеющие место при

$$
T \Delta=O(1), \quad \Lambda r / n \rightarrow 0, \quad \Lambda \rightarrow \infty
$$

и равенства (29), (32).

\section{2. Доказательства теорем}

Доказательство первой части теоремы 1. Это доказательство аналогично доказательству первой части теоремы 1 работы [1]. Положим

$$
N=\left|X_{r}^{\prime}\right|
$$

Занумеруем некоторым образом элементы множества $X_{r}^{\prime}$. Пусть

$$
X_{r}^{\prime}=\left\{x^{s}\right\}_{s=1}^{N}, \quad x^{s}=\left(x_{1}^{s}, \ldots, x_{n}^{s}\right)
$$

Представим $\xi$ в виде суммы индикаторов

$$
\xi=\xi_{1}+\ldots+\xi_{N},
$$

где

$$
\xi_{s}=I\left\{x^{s} \text { является решением системы }\right\}, \quad s=1, \ldots, N \text {. }
$$


Для факториальных моментов $\mathrm{E}(\xi)_{l}$ величины $\xi$ справедливо равенство

$$
\begin{aligned}
\mathbf{E}(\xi)_{l} & =\mathbf{E} \xi(\xi-1) \ldots(\xi-l+1) \\
& =\sum_{(s(1), \ldots, s(l))} b_{s(1) \ldots s(l),}
\end{aligned}
$$

где

$$
b_{s(1) \ldots s(l)}=\mathbf{P}\left\{\xi_{s(1)}=\xi_{s(2)}=\ldots=\xi_{s(l)}=1\right\}
$$

и суммирование производится по всем упорядоченным наборам $(s(1), \ldots, s(l))$ попарно различных индексов, принадлежащих множеству $\{1, \ldots, N\}$. Положим

$$
\begin{aligned}
M\left(x^{s}\right) & =\left\{i: I\left(x_{i}^{s}\right)=1\right\} \subseteq\{1, \ldots, n\}, \\
M\left(x^{s(1)}, \ldots x^{s(l)}\right) & =\bigcup_{j=1}^{l} M\left(x^{s(j)}\right), \\
\mu\left(x^{s(1)}, \ldots, x^{s(l)}\right) & =\left|M\left(x^{s(1)}, \ldots, x^{s(l)}\right)\right| .
\end{aligned}
$$
Пусть

Следуя работе [3] (см. также [4, 5]), введем исключительные множества $S_{l}, l=2,3, \ldots$

$$
S_{l}=\left\{(s(1), \ldots, s(l)): \mu\left(x^{s(1)}, \ldots, x^{s(l)}\right) \leqslant r l-r / 2\right\} .
$$

Покажем, что в условиях теоремы 1

$$
\max _{1 \leqslant s \leqslant N} b_{s} \rightarrow 0, \quad \sum_{s=1}^{N} b_{s} \rightarrow \lambda,
$$

и при любом $l=2,3 \ldots$

$$
\begin{aligned}
\sum_{(s(1), \ldots, s(l)) \in S_{l}} h_{s(1) \ldots s(l)} & \rightarrow 0 \\
\sum_{(s(1), \ldots, s(l)) \in S_{l}} h_{s(1)} h_{s(2)} \ldots h_{s(l)} & \rightarrow 0 \\
\max _{(s(1), \ldots, s(l)) \notin S_{l}}\left|\frac{h_{s(1) \ldots s(l)}}{h_{s(1)} \ldots h_{s(l)}}-1\right| & \rightarrow 0
\end{aligned}
$$

равномерно по $a \in G F(q)^{T}, a \neq 0$. Тогда воспользуемся теоремой 1 работы [3] и получим (12).

Соотношения (34)-(37) докажем с помощью ряда вспомогательных утверждений.

Пусть $x=\left(x_{1}, \ldots, x_{n}\right) \in X_{r}$. Положим

$$
\mathbf{P}\left\{\left(\varepsilon_{t}, x\right)=v\right\}=\frac{1}{q}\left(1+\Delta_{t}^{(v)}(x)\right),
$$

где

$$
\begin{aligned}
\varepsilon_{t} & =\left(\varepsilon_{t, 1}, \ldots, \varepsilon_{t, n}\right), \\
\left(\varepsilon_{t}, x\right) & =\varepsilon_{t, 1} x_{1}+\ldots+\varepsilon_{t, n} x_{n},
\end{aligned}
$$

при этом

$$
\sum_{\nu \in G F(q)} \Delta_{t}^{(\nu)}(x)=0
$$


Лемма 2. Для модуля $\Delta_{t}^{(v)}(x)$ справедлива оченка

$$
\left|\Delta_{t}^{(\nu)}(x)\right| \leqslant \Delta^{r}
$$

где $\Delta$ определяется формулами (6), (10).

Оценка (39) вытекает из формулы

$$
\Delta_{t}^{(v)}(x)=\frac{1}{q^{r-1}} \sum_{\substack{v_{1}, \ldots, \nu_{r} \in G F(q): k=1 \\ v_{1}+\ldots+v_{r}=v}} \prod_{k=1}^{r} \Delta_{t, i_{k}}^{v_{k} x_{i_{k}}^{-1}}
$$

где $x_{i_{1}}, \ldots, x_{i_{r}}-$ ненулевые координаты вектора $x$. Формула (40) доказывается с помощью индукции по натуральным значениям параметра $r$.

Используя (38), (39) получаем, что

$$
\begin{gathered}
q^{-T}\left(1-\Delta^{r}\right)^{T} \leqslant b_{s} \leqslant q^{-T}\left(1+\Delta^{r}\right)^{T}, \\
\Lambda\left(1-\Delta^{r}\right)^{T} \leqslant \sum_{s=1}^{N} b_{s} \leqslant \Lambda\left(1+\Delta^{r}\right)^{T} .
\end{gathered}
$$

Отсюда вытекают соотношения (34).

Осталось доказать соотношения (35), (36), (37).

Следующие вспомогательные утверждения являются близкими аналогами соответствующих лемм из работы [1]. С целью связности изложения доказательства большинства утверждений приводятся полностью.

Лемма 3. Пусть $1 \leqslant l \leqslant n$,

$$
\operatorname{rank}\left(x^{s(1)}, \ldots, x^{s(l)}\right)=m \leqslant l .
$$

Тогда при любом фиксированном $a=\left(a_{1}, \ldots, a_{T}\right)$

$$
b_{s(1) \ldots s(l)} \leqslant((1 / q)(1+\Delta))^{T m},
$$

при этом в случае $m=l$

$$
((1 / q)(1-\Delta))^{T l} \leqslant b_{s(1) \ldots s(l)} \leqslant((1 / q)(1+\Delta))^{T l} .
$$

Доказательство. Пусть

$$
\begin{aligned}
\xi_{s}^{(t)} & =I\left\{x^{s} \text { является решением уравнения с номером } t\right\}, \\
b_{s(1) \ldots s(l)}^{(t)} & =\mathbf{P}\left\{\xi_{s(1)}^{(t)}=\ldots=\xi_{s(l)}^{(t)}=1\right\} .
\end{aligned}
$$

В силу независимости векторов $\varepsilon_{t}=\left(\varepsilon_{t, 1}, \ldots, \varepsilon_{t, n}\right), t=1, \ldots, T$,

$$
b_{s(1) \ldots s(l)}=\prod_{t=1}^{T} b_{s(1) \ldots s(l)}^{(t)}
$$

Положим

$$
x^{s(j)}=\left(x_{1}^{s(j)}, \ldots, x_{n}^{s(j)}\right)
$$


и рассмотрим систему уравнений

$$
x_{1}^{s(j)} \varepsilon_{t, 1} \oplus \ldots \oplus x_{n}^{s(j)} \varepsilon_{t, n}=a_{t}, \quad j=1, \ldots, l,
$$

относительно переменных $\varepsilon_{t, 1}, \ldots, \varepsilon_{t, n}$. Пусть

$$
\operatorname{rank}\left(x^{s(1)}, \ldots, x^{s(l)}\right)=m \leqslant l<n .
$$

Без ограничения общности мы будем считать, что первые $m$ векторов столбцов $\left(x_{1}^{s(1)}, \ldots, x_{1}^{s(l)}\right), \ldots,\left(x_{m}^{s(1)}, \ldots, x_{m}^{s(l)}\right)$ матрицы коэффициентов системы (45) линейно независимы. Введем индикатор

$I_{t}\left(v_{m+1}, \ldots, v_{n}\right)=I$ ссистема (45) имеет решение с $\left.\varepsilon_{t, m+1}=v_{m+1}, \ldots, \varepsilon_{t, n}=v_{n}\right\}$.

Ясно, что

$$
b_{s(1) \ldots s(l)}^{(t)}=\sum_{v_{m+1}, \ldots, v_{n} \in G F(q), I_{t}\left(v_{m+1}, \ldots, v_{n}\right)=1} P\left(v_{m+1}, \ldots, v_{n}\right),
$$

где $P\left(v_{m+1}, \ldots, v_{n}\right)$ есть вероятность того, что значение случайного вектора $\left(\varepsilon_{t, 1}, \ldots, \varepsilon_{t, n}\right)$ совпадет с решением системы (45), в котором последние $n-m$ координат равны $v_{m+1}, \ldots, v_{n}$. Нетрудно установить, что

$$
P\left(v_{m+1}, \ldots, v_{n}\right) \leqslant((1 / q)(1+\Delta))^{m} \prod_{i=m+1}^{n}\left((1 / q)\left(1+\Delta_{t, i}^{\left(v_{i}\right)}\right)\right),
$$

и в случае $m=l$

$$
\begin{aligned}
((1 / q)(1-\Delta))^{m} \prod_{i=l+1}^{n}\left((1 / q)\left(1+\Delta_{t, i}^{\left(v_{i}\right)}\right)\right) & \leqslant P\left(v_{l+1}, \ldots, v_{n}\right) \\
& \leqslant((1 / q)(1+\Delta))^{m} \prod_{i=l+1}^{n}\left((1 / q)\left(1+\Delta_{t, i}^{\left(\nu_{i}\right)}\right)\right),
\end{aligned}
$$

при этом

$$
I_{t}\left(v_{l+1}, \ldots, v_{n}\right)=1, \quad\left(v_{l+1}, \ldots, v_{n}\right) \in G F(q)^{n-l} .
$$

Из (46), (47) и (46), (48), (49) получаем (43) и (44).

Лемма доказана.

Положим

$$
\rho\left(x^{s(1)}, \ldots, x^{s(l)}\right)=r l-\mu\left(x^{s(1)}, \ldots, x^{s(l)}\right) .
$$

Обозначим через $A\left(x^{s(1), \ldots, x^{s(l)}}\right)$ матрицу размера $l \times n$, составленную из строк $x^{s(1), \ldots, x^{s(l)}} \in X(r)=\left\{x^{s}\right\}_{s=1}^{N}$.

Лемма 4. Пусть при некоторых $\alpha_{1}, \ldots, \alpha_{k} \in G F(q), \alpha_{1}, \ldots, \alpha_{k} \neq 0$ имеет место равенство,

$$
\alpha_{1} x^{\left.s^{(} j_{1}\right)}+\ldots+\alpha_{k} x^{s\left(j_{k}\right)}=0
$$

Тогда

$$
\rho\left(x^{s\left(j_{1}\right)}, \ldots, x^{s\left(j_{k}\right)}\right) \geqslant r k / 2
$$


Доказательство. Отметим, что матрица $A\left(x^{s\left(j_{1}\right)}, \ldots, x^{s\left(j_{k}\right)}\right)$ содержит $r k$ ненулевых элементов, а число ненулевых столбцов в ней равно $\mu\left(x^{s\left(j_{1}\right)}, \ldots, x^{s\left(j_{k}\right)}\right)$. В силу $(51)$ каждый ненулевой столбец содержит не менее двух ненулевых элементов. Поэтому

$$
2 \mu\left(x^{s\left(j_{1}\right)}, \ldots, x^{s\left(j_{k}\right)}\right) \leqslant r k .
$$

Отсюда и из (50) следует (52).

Лемма 5. Пусть

$$
x^{s\left(j_{1}\right)}, \ldots, x^{s\left(j_{k}\right)} \in\left\{x^{s(1)}, \ldots, x^{s(l)}\right\} \subseteq X(r)
$$

Тогда

$$
\rho\left(x^{s(1)}, \ldots, x^{s(l)}\right) \geqslant \rho\left(x^{s\left(j_{1}\right)}, \ldots, x^{s\left(j_{k}\right)}\right)
$$

Доказательство. Положим

$$
\rho_{i}\left(x^{s(1)}, \ldots, x^{s(l)}\right)= \begin{cases}0, & \text { если } i \text {-й столбец матрицы } A\left(x^{s(1)}, \ldots, x^{(s(l)}\right) \\ v-1, & \text { содержит не более одного ненулевого элемента } \\ \text { если } i \text {-й столбец матрицы } A\left(x^{s(1)}, \ldots, x^{(s(l)}\right) \\ \text { содержит не менее двух ненулевых элементов. }\end{cases}
$$

Нетрудно видеть, что

$$
\rho\left(x^{s(1)}, \ldots, x^{s(l)}\right)=\sum_{i=1}^{n} \rho\left(x^{s(1)}, \ldots, x^{s(l)}\right)
$$

и при условии (45)

$$
\rho_{i}\left(x^{s(1)}, \ldots, x^{s(l)}\right) \geqslant \rho_{i}\left(x^{s\left(j_{1}\right)}, \ldots, x^{s\left(j_{k}\right)}\right), \quad i=1, \ldots, n .
$$

Отсюда и из (55) следует (54).

Лемма 6. Пусть набор $x^{s(1)}, \ldots, x^{s(l)}$ не содержит коллинеарных векторов,

$$
\mu\left(x^{s(1)}, \ldots, x^{s(l)}\right)>r(l-1)-r / 2 .
$$

Тогда

$$
\operatorname{rank}\left(x^{s(1)}, \ldots, x^{s(l)}\right)=l
$$

Доказательство. При $l=1$ и $l=2$ это утверждение очевидно. Пусть $l \geqslant 3$, выполняется (56) и

$$
\operatorname{rank}\left(x^{s(1)}, \ldots, x^{s(l)}\right)<l
$$

Тогда найдутся векторы

$$
x^{s\left(j_{1}\right)}, \ldots, x^{s\left(j_{k}\right)} \in\left\{x^{s(1)}, \ldots, x^{s(l)}\right\}, \quad k \geqslant 3,
$$

такие, что

$$
\alpha_{1} x^{s\left(j_{1}\right)}+\ldots+\alpha_{k} x^{s\left(j_{k}\right)}=0
$$


при некоторых $\alpha_{1}, \ldots, \alpha_{k} \in G F(q), \alpha_{1}, \ldots, \alpha_{k} \neq 0$. Поэтому согласно леммам 2 и 3

$$
\begin{aligned}
\rho\left(x^{s(1)}, \ldots, x^{s(l)}\right) & \geqslant \rho\left(z^{s\left(j_{1}\right)}, \ldots, x^{s\left(j_{k}\right)}\right) \\
& \geqslant \frac{r k}{2} \geqslant \frac{3 r}{2} .
\end{aligned}
$$

Отсюда получаем, что

$$
\begin{aligned}
\mu\left(x^{s(1)}, \ldots, x^{s(l)}\right) & =r l-\rho\left(x^{s(1)}, \ldots, x^{s(l)}\right) \\
& \leqslant r l-\frac{3 r}{2}=r(l-1)-\frac{r}{2} .
\end{aligned}
$$

Последнее неравенство противоречит неравенству (56). Значит, из (56) следует (57).

Лемма 7. Пусть $l \geqslant 3$,

$$
\operatorname{rank}\left(x^{s(1)}, \ldots, x^{s(l)}\right)<l
$$

и при некоторых $\alpha_{1}, \ldots, \alpha_{l-1}$ из $G F(q)$ таких, что

$$
\sum_{s=1}^{l-1} I\left(\alpha_{s}\right) \geqslant 2,
$$

имеет место равенство

$$
x^{s(l)}=\alpha_{1} x^{s(1)}+\ldots+\alpha_{l-1} x^{s(l-1)}
$$

Тогда

$$
\begin{aligned}
\mu\left(x^{s(1)}, \ldots, x^{s(l-1)}\right) & =\mu\left(x^{s(1)}, \ldots, x^{s(l)}\right) \\
\operatorname{rank}\left(x^{s(1)}, \ldots, x^{s(l-1)}\right) & =\operatorname{rank}\left(x^{s(1)}, \ldots, x^{s(l)}\right) .
\end{aligned}
$$

Доказательство. Из (58) вытекает, что столбцы матрицы $A\left(x^{s(1)}, \ldots, x^{s(l)}\right)$, соответствующие ненулевым элементам вектора $x^{s(l)}$, содержат не менее двух ненулевых элементов. Поэтому вычеркивание строки $x^{s(l)}$ из матрицы $A\left(x^{s(1)}, \ldots, x^{s(l)}\right)$ не изменяет общего числа ненулевых столбцов. Отсюда следует (59). Равенство (60) очевидно.

Лемма 8. Пусть $l \geqslant 3$, набор $x^{s(1)}, \ldots, x^{s(l)}$ не содержит коллинеарных векторов,

$$
\mu\left(x^{s(1)}, \ldots, x^{s(l)}\right)>r(m-1)-r / 2,
$$

где $m$ - целое число, $2 \leqslant m \leqslant l$.

Тогда

$$
\operatorname{rank}\left(x^{s(1)}, \ldots, x^{s(l)}\right) \geqslant m
$$

Доказательство. В случае $m=l$ это утверждение вытекает из леммы 6 , а в случае $m=2$ оно тривиально. Пусть

$$
2<m<l, \quad \operatorname{rank}\left(x^{s(1)}, \ldots, x^{s(l)}\right)<m .
$$


Применяя $l-m$ раз лемму 5 , установим, что найдутся векторы $x^{s(1)}, \ldots, x^{s(m)}$ (без ограничения общности будем полагать, что эти векторы первые) такие, что

$$
\begin{aligned}
\mu\left(x^{s(1)}, \ldots, x^{s(m)}\right) & =\mu\left(x^{s(1)}, \ldots, x^{s(l)}\right), \\
\operatorname{rank}\left(x^{s(1)}, \ldots, x^{s(m)}\right) & =\operatorname{rank}\left(x^{s(1)}, \ldots, x^{s(l)}\right) .
\end{aligned}
$$

Из (61), (64) и леммы 4 следует, что

$$
\operatorname{rank}\left(x^{s(1)}, \ldots, x^{s(l)}\right)=m .
$$

Это равенство противоречит предположению (63). Значит, из (61) следует (62).

Лемма доказана.

Лемма 9. Пусть

$$
a=\left(a_{1}, \ldots, a_{T}\right) \neq(0, \ldots, 0)
$$

и набор $x^{s(1)}, \ldots, x^{s(l)}, l \geqslant 2$, содержит коллинеарные векторы. Тогда

$$
b_{s(1)} \ldots s(l)=0 .
$$

Доказательство. Данное утверждение вытекает из очевидного факта, что два коллинеарных вектора не могут оба удовлетворять одному уравнению с правой частью, отличной от нуля.

Лемма доказана.

Пусть

$$
a=\left(a_{1}, \ldots, a_{T}\right) \neq(0, \ldots, 0) .
$$

Обозначим $W_{l}(h)$ число упорядоченных наборов $(s(1), \ldots, s(l))$ попарно различных индексов таких, что

$$
\mu\left(x^{s(1)}, \ldots, x^{s(l)}\right)=h .
$$

Используя леммы $3,8,9$, получаем оценку

$$
\begin{aligned}
\sum_{(s(1), \ldots, s(l))} b_{(s(1) \ldots s(l)) \in S_{l}} \leqslant((1 / q)(1+\Delta))^{2 T} & \sum_{r+1 \leqslant h \leqslant 2 r-r / 2} W_{l}(h) \\
& +\sum_{m=3}^{l}((1 / q)(1+\Delta))^{m T} \sum_{r(m-1)-r / 2<h \leqslant r m-r / 2} W_{l}(h),
\end{aligned}
$$

где $l \geqslant 2$ и в случае $l=2$ правая часть неравенства (65) содержит только первое слагаемое. Нетрудно установить, что

$$
W_{l}(h) \leqslant\left(\begin{array}{l}
n \\
h
\end{array}\right)\left(\begin{array}{l}
h \\
r
\end{array}\right)^{l}(q-1)^{r l} .
$$

Пусть $\omega=\lfloor r / 2\rfloor$ - целая часть числа $r / 2$. Из (65), (66) вытекает, что

$$
\sum_{(s(1), \ldots, s(l)) \in S_{l}} b_{(s(1) \ldots s(l)} \leqslant R_{l},
$$


где

$$
\begin{aligned}
R_{l} & =(1+\Delta)^{l T} \sum_{m=2}^{l} R_{l}^{(m)}(\omega), \\
R_{l}^{(m)}(\omega) & =r q^{-m T}\left(\begin{array}{c}
n \\
r m-\omega
\end{array}\right)\left(\begin{array}{c}
r m-\omega \\
r
\end{array}\right)^{l}(q-1)^{r l} .
\end{aligned}
$$

При этом в силу (2), (9)

$$
R_{l}^{(m)}(\omega) \leqslant r \Lambda^{m}\left(\begin{array}{l}
n \\
r
\end{array}\right)^{-m}\left(\begin{array}{c}
n \\
r m-\omega
\end{array}\right)\left(\begin{array}{c}
r m-\omega \\
r
\end{array}\right)^{l}(q-1)^{r l} .
$$

Лемма 10. Найдется постоянная $C<\infty$ такая, что

$$
R_{l}^{(m)}(\omega) \leqslant \Lambda^{m} \exp \{-\omega \ln (n / r)+r l(\ln l+C)\}
$$

при всех $n, r, l, r l / n \leqslant 1 / 2,2 \leqslant m \leqslant l$.

Данное утверждение вытекает из леммы 7 работы [1], где дана оценка вида (71) для правой части неравенства (70) в случае $q=2$, то есть при отсутствии множителя $(q-1)^{r l}$. Ясно, что появление этого множителя не меняет вида оценки (71) (за счет постоянной $C$ ).

С учетом (71) получаем, что

$$
\begin{aligned}
R_{l} & =(1+\Delta)^{l T} \sum_{m=2}^{l} R_{l}^{(m)}(\lfloor r / 2\rfloor) \\
& \leqslant(1+\Delta)^{l T} \frac{\Lambda^{l}-1}{\Lambda-1} \exp \{-\lfloor r / 2\rfloor \ln (n / r)+r l(\ln l+C)\},
\end{aligned}
$$

где при условиях теоремы 1 правая часть неравенства стремится к нулю, так как

$$
(1+\Delta)^{l T} \leqslant \exp \{l T \Delta\}
$$

и в силу неравенств

$$
\left(\begin{array}{l}
n \\
r
\end{array}\right)(1-q)^{r} q^{-T} \geqslant \Lambda \geqslant C^{\prime \prime}>0,
$$

где $C^{\prime \prime}$ - постоянная величина, имеют место оценки

$$
\begin{aligned}
T & =O(r \ln (n / r)), \\
T \Delta & =o(r \ln (n / r)) .
\end{aligned}
$$

Таким образом, из (67) получаем (35).

Аналогично доказывается (36). Действительно,

$$
b_{s(1)} b_{s(2)} \ldots b_{s(l)} \leqslant((1 / q)(1+\Delta))^{l T},
$$

и так же как (65), получаем, что

$$
\begin{aligned}
& \sum_{(s(1), \ldots, s(l)) \in S_{l}} b_{s(1)} b_{s(2)} \ldots b_{s(l)} \\
& \quad \leqslant((1 / q)(1+\Delta))^{l T}\left(\sum_{r+1 \leqslant h \leqslant 2 r-r / 2} W_{l}(h)+\sum_{m=3}^{l} \sum_{r(m-1)-r / 2<h \leqslant r m-r / 2} W_{l}(h)\right) .
\end{aligned}
$$


Следовательно, имеет место оценка

$$
\sum_{(s(1), \ldots, s(l)) \in S_{l}} b_{s(1)} b_{s(2)} \ldots b_{s(l)} \leqslant R_{l}
$$

аналогичная (67). При этом, как показано выше, $R_{l} \rightarrow 0$.

Осталось показать, что

$$
\left|b_{s(1) \ldots s(l)} /\left(b_{s(1)} \ldots b_{s(l)}\right)-1\right| \rightarrow 0
$$

при условии, что

$$
r l-r / 2<\mu(s(1), \ldots, s(l)), \quad l \geqslant 2 .
$$

Положим

$$
\begin{aligned}
\varepsilon & =\left(\varepsilon_{1}, \ldots, \varepsilon_{n}\right), \\
x^{s(j)} & =\left(x_{1}^{s(j)}, \ldots, x_{n}^{s(j)}\right), \\
\left(\varepsilon, x^{s(j)}\right) & =\varepsilon_{1} x_{1}^{s(j)}+\ldots+\varepsilon_{n} x_{n}^{s(j)}
\end{aligned}
$$

и обозначим $P\left(x^{s(1)}, \ldots, x^{s(l)} ; \gamma_{1}, \ldots, \gamma_{l}\right)$ вероятность события

$$
\left\{\left(\varepsilon, x^{s(j)}\right)=\gamma_{j}, j=1, \ldots, l\right\}
$$

при случайных и независимых $\varepsilon_{1}, \ldots, \varepsilon_{n}$ таких, что

$$
\mathbf{P}\left\{\varepsilon_{i}=v\right\}=\left(1+\Delta_{i}^{\nu}\right) / q, \quad v \in G F(q), \quad\left|\Delta_{i}^{(v)}\right| \leqslant \Delta<1, \quad i=1, \ldots, n .
$$

Здесь $\gamma_{1}, \ldots, \gamma_{l}$ - произвольные фиксированные элементы поля $G F(q)$.

Лемма 11. Пусть выполняется условие (76). Тогда при $\Delta \rightarrow 0$

$$
P\left(x^{s(1)}, \ldots, x^{s(l)} ; \gamma_{1}, \ldots, \gamma_{l}\right)=q^{-l}\left(1+O\left(\Delta^{r}\right)\right)
$$

равномерно по $\gamma_{1}, \ldots, \gamma_{l} \in G F(q)$.

Доказательство. Введем в рассмотрение множества

$$
\begin{array}{rlr}
A_{j} & =\left\{i: I\left(x_{i}^{s(j)}\right)=1\right\}, & j \in\{1, \ldots, l\}, \\
A_{j_{1}, \ldots, j_{k}} & =\bigcap_{\nu=1}^{k} A_{j_{1}}, & j_{1}, \ldots, j_{k} \in\{1, \ldots, l\}, \\
B_{j_{1}, \ldots, j_{k}} & =\bigcup_{j \in\{1, \ldots, l\} \backslash\left\{j_{1}, \ldots, j_{k}\right\}} A_{j}, & B_{1, \ldots, l}=\varnothing, \\
C_{j_{1}, \ldots j_{k}} & =A_{j_{1}, \ldots, j_{k}} \backslash\left(A_{j_{1}, \ldots, j_{k}} \cap B_{j_{1}, \ldots, j_{k}}\right) . &
\end{array}
$$

Согласно определению, соответствующие различным наборам $j_{1}, \ldots, j_{k}$ множества $C_{j_{1}, \ldots, j_{k}}$ не содержат общих элементов, при этом

$$
A_{j}=C_{j} \cup D(j),
$$


где

$$
D(j)=\bigcup_{k=2}^{l} \bigcup_{\left(j_{1}, \ldots, j_{k}\right) \in M_{j}^{k}} C_{j_{1}, \ldots, j_{k}}
$$

$M_{j}^{k}$ - множество упорядоченных наборов индексов из $\{1, \ldots, l\}$ объема $k \leqslant l$, содержащих фиксированный индекс $j \in\{1, \ldots, l\}$.

С учетом равенств

$$
\begin{aligned}
r l & =\sum_{j=1}^{l}\left|C_{j}\right|+\sum_{k=2}^{l} k \sum_{1 \leqslant j_{1}<\ldots<j_{k} \leqslant l}\left|C_{j_{1} \ldots j_{k}}\right|, \\
\mu(s(1), \ldots, s(l)) & =\sum_{j=1}^{l}\left|C_{j}\right|+\sum_{k=2}^{l} \sum_{1 \leqslant j_{1}<\ldots<j_{k} \leqslant l}\left|C_{j_{1} \ldots j_{k}}\right|
\end{aligned}
$$

условие (76) принимает вид

$$
r l-\mu(s(1), \ldots, s(l))=\sum_{k=2}^{l}(k-1) \sum_{1 \leqslant j_{1}<\ldots<j_{k} \leqslant l}\left|C_{j_{1} \ldots j_{k}}\right|<r / 2 .
$$

Покажем, что при условии (81) ((76))

$$
\left|C_{j}\right|>r / 2, \quad j=1, \ldots, l .
$$

Действительно, пусть существует $j \in\{1, \ldots, l\}$ такое, что $\left|C_{j}\right| \leqslant r / 2$. Тогда в силу (79) $|D(j)| \geqslant r / 2$, что противоречит условию (81) ((76)), так как согласно (80) для всех $j \in\{1, \ldots, l\}$

$$
\sum_{k=2}^{l}(k-1) \sum_{1 \leqslant j_{1}<\ldots<j_{k} \leqslant l}\left|C_{j_{1} \ldots j_{k}}\right| \geqslant|d(j)| .
$$

Следовательно, при условии (81) ((76)) имеет место (82).

Положим

$$
\begin{aligned}
x^{j} & =\left(x_{1}^{j}, \ldots, x_{n}^{j}\right), \\
x^{j_{1}, \ldots, j_{k}} & =\left(x_{1}^{j_{1}, \ldots, j_{k}}, \ldots, x_{n}^{j_{1}, \ldots, j_{k}}\right), \\
\Gamma_{\alpha}(j) & =\sum_{k=2}^{l} \sum_{\left(j_{1}, \ldots, j_{k}\right) \in M_{j}^{k}}\left(\alpha, x^{s(j)} x^{j_{1}, \ldots, j_{k}}\right),
\end{aligned}
$$

где

$$
\begin{aligned}
x_{i}^{j} & = \begin{cases}1, & i \in C_{j}, \\
0, & i \notin C_{j},\end{cases} \\
x_{i}^{j_{1}, \ldots, j_{k}} & = \begin{cases}1, & i \in C_{j_{1}, \ldots, j_{k},}, \\
0, & i \notin C_{j_{1}, \ldots, j_{k}},\end{cases} \\
\alpha & =\left(\alpha_{1}, \ldots, \alpha_{n}\right) \in(G F(q))^{n}, \\
x^{s(j)} x^{j_{1}, \ldots, j_{k}} & =\left(x_{1}^{s(j)} x_{1}^{j_{1}, \ldots, j_{k}}, \ldots, x_{n}^{s(j)} x_{n}^{j_{1}, \ldots, j_{k}}\right) \in(G F(q))^{n}, \\
\left(j_{1}, \ldots, j_{k}\right) & \in M_{j}^{k}, \quad j \in\{1, \ldots, l\}, \quad i \in\{1, \ldots n\},
\end{aligned}
$$


и операции в выражениях (83), (84) производятся в поле $G F(q)$. Пусть

$$
D=\bigcup_{k=2}^{l} \bigcup_{1 \leqslant j_{1}<\ldots<j_{k} \leqslant l} C_{j_{1}, \ldots, j_{k}} .
$$

Представим событие (77) в виде суммы попарно несовместных событий

$$
\bigcap_{i \in D}\left\{\varepsilon_{i}=\alpha_{i}\right\} \bigcap_{j=1}^{l}\left\{\left(\varepsilon, x^{s(j)} x^{j}\right)=\gamma_{j}-\Gamma_{\alpha}(j)\right\},
$$

взятых для значений $\alpha_{i}, i \in D$ (здесь учитывается, что входящая в выражение (86) величина (83), индексированная вектором $\alpha=\left(\alpha_{1}, \ldots, \alpha_{n}\right)$, зависит только от координат $\left.\alpha_{i}, i \in D\right)$.

Используя вышеуказанное представление и полагая

$$
\mathbf{P}\left\{\left(\varepsilon, x^{s(j)} x^{j}\right)=\gamma_{j}-\Gamma_{\alpha}(j)\right\}=(1 / q)\left(1+\Delta^{\left(\gamma_{j}-\Gamma_{\alpha}(j)\right)}\left(x^{s(j)} x^{j}\right)\right),
$$

получаем, что

$$
\begin{aligned}
P\left(x^{s(1)}, \ldots, x^{s(l)} ; \gamma_{1}, \ldots, \gamma_{l}\right) & \\
& =\sum_{\alpha_{i} \in G F(q), i \in D} \prod_{i \in d} \frac{1}{q}\left(1+\Delta_{i}^{\left(\alpha_{i}\right)}\right) \prod_{j=1}^{l} \frac{1}{q}\left(1+\Delta^{\left(\gamma_{j}-\Gamma_{\alpha}(j)\right)}\left(x^{s(j)} x^{j}\right)\right) .
\end{aligned}
$$

Раскрывая (87), находим, что

$$
\begin{aligned}
& q^{l} P\left(x^{s(1)}, \ldots, x^{s(l)} ; \gamma_{1}, \ldots, \gamma_{l}\right) \\
& =\sum_{\alpha_{i} \in G F(q), i \in D} \prod_{i \in D} \frac{1}{q}\left(1+\Delta_{i}^{\left(\alpha_{i}\right)}\right)\left(1+\sum_{j=1}^{l} \Delta^{\left(\gamma_{j}-\Gamma_{\alpha}(j)\right)}\left(x^{s(j)} x^{j}\right)\right. \\
& \left.\quad+\sum_{k=2}^{l} \sum_{1 \leqslant j_{1}<\ldots<j_{k} \leqslant l} \Delta^{\left(\gamma_{j_{1}}-\Gamma_{\alpha}\left(j_{1}\right)\right)}\left(x^{\left(j_{1}\right)} x^{j_{1}}\right) \ldots \Delta^{\left(\gamma_{j_{k}}-\Gamma_{\alpha}\left(j_{k}\right)\right)}\left(x^{\left(j_{k}\right)} x^{j_{k}}\right)\right) \\
& =1+\sum_{j=1}^{l} \Delta^{\left(\gamma_{j}\right)}\left(x^{s(j)}\right)+O\left(m^{2}\right),
\end{aligned}
$$

где

$$
m=\max _{j \in\{1, \ldots, l\}, \gamma \in G F(q)}\left|\Delta^{(\gamma)}\left(x^{s(j)} x^{j}\right)\right|
$$

Согласно оценке (39)

$$
\left|\Delta^{\left(\gamma_{j}\right)}\left(x^{s(j)}\right)\right| \leqslant \Delta^{r}, \quad j=1, \ldots, l
$$

Вместе с этим

$$
m \leqslant \max _{j \in\{1, \ldots, l\}} \Delta^{\left|C_{j}\right|} \leqslant \Delta^{r / 2} .
$$

Теперь из (88)-(91) получаем (78).

Лемма 11 доказана. 
Из леммы 11 следует, что при условии (76)

$$
b_{s(1) \ldots s(l)}=(1 / q)^{T l}\left(1+O\left(\Delta^{r}\right)\right)^{T}
$$

равномерно по значениям вектора правых частей $a$. Вместе с этим

$$
\left((1 / q)\left(1-\Delta^{r}\right)\right)^{T l} \leqslant b_{s(1)} \ldots b_{s(l} \leqslant\left((1 / q)\left(1+\Delta^{r}\right)\right)^{T l} .
$$

Из (92), (93) следует (75). Первая часть теоремы 1 доказана.

Доказательство второй части теоремы 1. Пусть подмножество $X_{r}^{\prime} \subseteq X_{r}$ состоит из представителей, взятых по одному от каждого класса коллинеарных векторов, на которые разбивается множество $X_{r}$. При этом

$$
\left|X_{r}^{\prime}\right|=\left|X_{r}\right| /(q-1)=\left(\begin{array}{l}
n \\
r
\end{array}\right)(q-1)^{r-1} .
$$

Если вектор $x^{*}$ является решением системы $E x=0, E=\left\|\varepsilon_{t, i}\right\|$, с ограничением $x \in X_{r}$, то решением этой системы является и любой коллинеарный с ним вектор $\beta x^{*}=\left(\beta x_{1}^{*}, \ldots, \beta x_{n}^{*}\right), \beta \in G F(q), \beta \neq 0$. Поэтому число решений $\xi$ системы $E x=0$ с ограничением $x \in X_{r}$ и число решений $\xi^{\prime}$ системы $E x=0$ с ограничением $x \in X_{r}^{\prime}$ связаны равенством

$$
\xi=\xi^{\prime}(q-1)
$$

Рассмотрим распределение величины $\xi^{\prime}$. Полагая $a=0$ и повторяя за исключением леммы 11 все рассуждения и выкладки, использованные при доказательстве первой части теоремы 1 , получаем, что $\xi^{\prime} \rightarrow \Pi(\lambda /(1-q))$. Следовательно,

$$
\xi(1-q)^{-1} \rightarrow \Pi(\lambda /(1-q))
$$

Вторая часть теоремы 1 доказана.

Доказательство третьей части теоремы 1 повторяет доказательство первой части теоремы 1 после исключения леммы 9 и требования $a \neq 0$.

Доказательство теоремы 2. В случае, когда подмножество $X_{r}^{\prime}$ определяется формулой (3), $|B|=1, B=\{\beta\}$, будем полагать

$$
\xi=f_{\beta}(E, a \mid r)
$$

Лемма 12. Пусть подмножество $X_{r}^{\prime}$ определяется формулой (3), $|B|=1, B=\{\beta\}, \beta-$ произвольный элемент $G F(q) \backslash\{0\}$, вектор а распределен равномерно. Тогда

$$
\begin{array}{ll}
\mathbf{P}\left\{f_{\beta}(E, a \mid r)=k\right\}=\mathbf{P}\left\{f_{\delta}(E, a \mid r)\right\}=k, & k=0,1, \ldots, \\
\mathbf{P}\left\{f_{\beta}(E, a \mid r)=k\right\}=\mathbf{P}\left\{f_{\delta}(E, a \mid n-r)\right\}=k, & k=0,1, \ldots,
\end{array}
$$

где $\delta$ - произвольный элемент $G F(q) \backslash\{0\}$. 
Доказательство. Так как (96) следует из (97), достаточно доказать равенства (97). Пусть некоторая реализация $\left(E^{\prime}, a^{\prime}\right)$ системы $E x=a$ имеет решение

$$
x^{\prime}=\left(x_{1}^{\prime}, \ldots, x_{n}^{\prime}\right), \quad \sum_{i=1}^{n} I_{\beta}\left(x_{i}^{\prime}\right)=r, \quad \sum_{i=1}^{n} I_{0}\left(x_{i}^{\prime}\right)=n-r
$$

где

$$
I_{\beta}= \begin{cases}1, & x_{i}^{\prime}=\beta \\ 0, & x_{i}^{\prime} \neq \beta\end{cases}
$$

Тогда реализация $\left(E^{\prime}, \alpha^{\prime}-\varepsilon^{\prime}\right)$, где

$$
\begin{aligned}
\alpha^{\prime}-\varepsilon^{\prime} & =\left(\alpha_{1}^{\prime}-\varepsilon_{1}^{\prime}, \ldots, \alpha_{T}^{\prime}-\varepsilon_{T}^{\prime}\right), \\
\varepsilon_{t}^{\prime} & =\beta\left(\varepsilon_{t, 1}^{\prime}+\ldots+\varepsilon_{t, n}^{\prime}\right),
\end{aligned}
$$

имеет решение

$$
x^{\prime \prime}=\left(x_{1}^{\prime \prime}, \ldots, x_{n}^{\prime \prime}\right)=\left(x_{1}^{\prime}-\beta, \ldots, x_{n}^{\prime}-\beta\right), \quad \sum_{i=1}^{n} I_{-\beta}\left(x_{i}^{\prime \prime}\right)=n-r, \quad \sum_{i=1}^{n} I_{0}\left(x_{i}^{\prime \prime}\right)=r .
$$

Отсюда вытекает, что

$$
f_{\beta}\left(E^{\prime}, \alpha \mid r\right)=f_{-\beta}\left(E^{\prime}, \alpha^{\prime}-\varepsilon^{\prime} \mid n-r\right) .
$$

Вместе с этим, при любом $\delta \in G F(q) \backslash\{0\}$

$$
f_{\beta}\left(E^{\prime}, \alpha \mid r\right)=f_{\delta}\left(E^{\prime}, \alpha \beta^{-1} \delta \mid r\right),
$$

где

$$
\alpha^{\prime} \beta^{-1} \delta=\left(\alpha^{\prime} \beta^{-1} \delta, \ldots, \alpha_{T}^{\prime} \beta^{-1} \delta\right) .
$$

Из (98), (99) вытекает, что

$$
f_{\beta}\left(E^{\prime}, \alpha^{\prime} \mid r\right)=f_{\delta}\left(E^{\prime},\left(\alpha^{\prime}-\varepsilon^{\prime}\right)(-\beta)^{-1} \delta \mid n-r\right) .
$$

При этом в силу равномерности распределения вектора $a$

$$
\mathbf{P}\left\{(E, a)=\left(E^{\prime}, \alpha^{\prime}\right)\right\}=\mathbf{P}\left\{(E, a)=\left(E^{\prime}, \alpha^{\prime \prime}\right)\right\}
$$

при любых $\alpha^{\prime}, \alpha^{\prime \prime} \in G F(q)^{T}$.

Используя (100), (101), получаем, что

$$
\begin{aligned}
\mathbf{P}\left\{f_{\beta}(E, a \mid r)=k\right\} & =\sum_{E^{\prime}, \alpha^{\prime}: f_{\beta}\left(E^{\prime}, \alpha^{\prime} \mid r\right)=k} \mathbf{P}\left\{(E, a)=\left(E^{\prime}, \alpha^{\prime}\right)\right\} \\
& =\sum_{E^{\prime}, \alpha^{\prime}: f_{\delta}\left(E^{\prime},\left(\alpha^{\prime}-\varepsilon^{\prime}\right)(-\beta)^{-1} \delta \mid n-r\right)=k} \mathbf{P}\left\{(E, a)=\left(E^{\prime},\left(\alpha^{\prime}-\varepsilon^{\prime}\right)(-\beta)^{-1} \delta\right)\right\} \\
& =\sum_{E^{\prime}, \alpha^{\prime \prime}: f_{\delta}\left(E^{\prime} \alpha^{\prime \prime} \mid n-r\right)=k} \mathbf{P}\left\{(E, a)=\left(E^{\prime}, \alpha^{\prime \prime}\right)\right\} \\
& =\mathbf{P}\left\{f_{\delta}(E, a \mid n-r)=k\right\}, \quad k=0,1, \ldots
\end{aligned}
$$

Лемма 12 доказана. 
Поскольку множество $X_{r}^{\prime}$, заданное формулой (3), где $|B|=1$, не содержит коллинеарных векторов, теорема 2 является следствием третьей части теоремы 1 и равенств (97), где следует заменить $\delta$ символом $\beta$. Теорема 2 доказана.

Доказательство теоремы 3. Пусть $x^{\prime}, x^{\prime \prime}$ - произвольные векторы из множества $X_{r}^{\prime}$. Нетрудно заметить, что существует $n \times n$ матрица $\pi$ над полем $G F(q)$, содержащая в каждой строке и в каждом столбце по одному ненулевому элементу и такая, что

$$
\begin{aligned}
x^{\prime \prime} & =\pi x^{\prime}, \\
g\left(E \mid x^{\prime}, r\right) & =g\left(E \pi^{-1} \mid x^{\prime \prime}, r\right) .
\end{aligned}
$$

В силу условий теоремы

$$
\mathbf{P}\left\{E=E^{\prime}\right\}=\mathbf{P}\left\{E=E^{\prime} \pi^{-1}\right\} .
$$

Действительно, при условии (а) теоремы 3 равенство (103) очевидно, а при (б) оно вытекает из того, что $\pi$ является подстановочной $(0,1)$-матрицей и $E^{\prime} \pi^{-1}$ получается из матрицы $E^{\prime}$ перестановкой столбцов.

Используя (102), (103), получаем, что

$$
\begin{aligned}
\mathbf{P}\left\{g\left(E \mid x^{\prime}, r\right)=k\right\} & =\sum_{E^{\prime}: g\left(E^{\prime} \mid x^{\prime \prime}, r\right)=k} \mathbf{P}\left\{E=E^{\prime}\right\} \\
& =\sum_{E^{\prime}: g\left(E^{\prime} \pi^{-1} \mid x^{\prime \prime}, r\right)=k} \mathbf{P}\left\{E=E^{\prime} \pi^{-1}\right\} \\
& =\sum_{E^{\prime \prime}: g\left(E^{\prime \prime} \mid x^{\prime \prime}, r\right)=k} \mathbf{P}\left\{E=E^{\prime \prime}\right\}=\mathbf{P}\left\{g\left(E \mid x^{\prime \prime}, r\right)=k\right\} .
\end{aligned}
$$

Теорема 3 доказана.

Доказательство теоремы 4. Приведем доказанное ранее вспомогательное утверждение (см. лемму 11 в [1] и теорему 4.1 в [2]).

Лемма 13. Пусть распределение числа $\eta=g\left(E \mid x^{0}, r\right)$ инвариантно относительно $x^{0} \in X_{r}^{\prime}$ и в системе уравнений с независимыми левыми и правыми частями вектор правых частей а распределен равномерно. Тогда

$$
\mathbf{P}\{\xi=k\}=\frac{1}{k} \mathbf{E} \xi \mathbf{P}\{\eta=k\}, \quad k=1,2, \ldots
$$

Используя теорему 3, лемму 13 и следствие 2, получаем (15). Теорема 4 доказана.

Доказательство теоремы 5. В случае, когда подмножество $X_{r}^{\prime}$ определяется формулой (3), $|B|=1, B=\{\beta\}, \beta \in G F(q) \backslash\{0\}$, будем полагать

$$
\begin{aligned}
X_{r}^{\prime} & =X_{r}(\beta), \\
\eta & =g_{\beta}\left(E \mid x^{0}, r\right) .
\end{aligned}
$$

Лемма 14. Справедливы соотношения

$$
\begin{aligned}
& g_{\beta}\left(E \mid x^{0}, r\right)=g_{\delta}\left(E \mid x^{\prime}, r\right), \\
& g_{\beta}\left(E \mid x^{0}, r\right)=g_{\delta}\left(E \mid x^{\prime \prime}, n-r\right),
\end{aligned}
$$


где $\delta$ - произвольный элемент $G F(q) \backslash\{0\}$,

$$
\begin{aligned}
& x^{0}=\left(x_{1}^{0}, \ldots, x_{1}^{0}\right) \\
& x^{\prime \prime}=\left(\left(\beta-x_{1}^{0}\right) \beta^{-1} \delta, \ldots,\left(\beta-x_{n}^{0}\right) \beta^{-1} \delta\right) .
\end{aligned}
$$

Доказательство. Определим $n$-мерные векторы

$$
\begin{aligned}
\bar{\beta} & =(\beta, \ldots, \beta), \\
x \alpha & =\left(x_{1} \alpha, \ldots, x_{n} \alpha\right), \\
\bar{\beta}-x & =\left(\beta-x_{1}, \ldots, \beta-x_{n}\right),
\end{aligned}
$$

где $\beta, \alpha, x_{i} \in G F(q)$. Очевидно, что равенство

$$
E x=E x^{0}, \quad x, x^{0} \in X_{r}(\beta),
$$

выполняется, если и только если выполняется равенство

$$
E x \beta^{-1} \delta=E x^{\prime}, \quad x \beta^{-1} \delta, \quad x^{\prime} \in X_{r}(\delta) .
$$

Отсюда вытекает (104).

Аналогично, равенство

$$
E x=E x^{0}, \quad x, x^{0} \in X_{r}(\beta),
$$

выполняется, если и только если выполняется равенство

$$
E(\bar{\beta}-x) \beta^{-1} \delta=E x^{\prime \prime}, \quad(\bar{\beta}-x) \beta^{-1} \delta, \quad x^{\prime \prime} \in X_{n-r}(\delta) .
$$

Отсюда вытекает (105). Лемма доказана.

Теорема 5 вытекает из теоремы 4 и равенства (105).

Доказательство теоремы 6. Доказательство первой части теоремы по сути совпадает с доказательством первой части теоремы 3 из работы [1] и отличается только нумерацией используемых ссылок. Для полноты изложения приведем его.

Пусть некоторая случайная величина $\zeta$ представляется суммой индикаторов

$$
\zeta=\zeta_{1}+\ldots+\zeta_{N}
$$

где $\zeta_{k}, k=1, \ldots, N$, принимают лишь значения 1,0 . Положим

$$
b_{s(1) \ldots s(l)}=\mathbf{P}\left\{\zeta_{s(1)}=\ldots=\zeta_{s(l)}=1\right\}
$$

где $s(1), \ldots, s(l) \in\{1, \ldots, N\}$.

Лемма 15 ([1]). Пусть при $N \rightarrow \infty$ выполняются условия

(1) $\hat{\Lambda}=\sum_{s=1}^{N} b_{s} \rightarrow \infty$,

(2) $\Delta_{l}=\sum_{(s(1), \ldots, s(l))}\left(b_{s(1) \ldots s(l)}-b_{s(1)} \ldots b_{s(l)}\right)=o\left(\hat{\Lambda}^{-t}\right), l=2,3, \ldots, t=1,2, \ldots$, 
(3) существует предел

$$
\lim _{N \rightarrow \infty} \frac{1}{\hat{\Lambda}} \sum_{s=1}^{N} b_{s}^{2}=\gamma
$$

Тогда распределение величины $(\zeta-\hat{\Lambda}) / \hat{\Lambda}^{1 / 2}$ слабо сходится к нормальному распределению с нулевым средним и дисперсией $\sigma^{2}=1-\gamma$.

Проверим условия леммы 15. В силу (41), (42) условия (1), (3), очевидно, выполняются, при этом

$$
\frac{1}{\hat{\Lambda}} \sum_{s=1}^{N} b_{s}^{2} \rightarrow 0 .
$$

Проверим условие (2). Пусть $a \neq 0$. С учетом (67), (74) находим, что

$$
\Sigma_{l}^{(1)}=\sum_{(s(1), \ldots, s(l)) \in S_{l}}\left|b_{s(1) \ldots s(l)}-b_{s(1)} \ldots b_{s(l)}\right| \leqslant 2 R_{l},
$$

где $R_{l}$ - величина, заданная формулами (68), (69). Используя (71), получаем, что при условии (17)

$$
\Sigma_{l}^{(1)}=o\left(\hat{\Lambda}^{-t}\right), \quad l=2,3, \ldots, \quad t=1,2, \ldots
$$

Рассмотрим теперь суммы

$$
\Sigma_{l}^{(2)}=\sum_{r l-r / 2<\mathbf{E} u(s(1), \ldots, s(l))}\left|b_{s(1) \ldots s(l)}-b_{s(1)} \ldots b_{s(l)}\right| .
$$

Согласно оценке (92) (которая выполняется при условии (76)) и согласно неравенствам (93)

$$
\left|h_{s(1) \ldots s(l)} / h_{s(1)} \ldots h_{s(l)}-1\right|=O\left(T \Delta^{r}\right) .
$$

Отсюда следует, что при условии (18)

$$
\Sigma_{l}^{(2)}=o\left(\hat{\Lambda}^{-t}\right), \quad l=2,3, \ldots, \quad t=1,2, \ldots
$$

Таким образом, все условия леммы 15 выполняются, причем равномерно по $a \neq 0$. Используя лемму 15 , оценку

$$
\hat{\Lambda}=\Lambda\left(1+O\left(T \Delta^{r}\right)\right)
$$

и соотношение (18), получаем (12).

Замечание 3. Доказательство леммы 15 в [1] следует дополнить следующим пояснением. Приведенное в доказательстве соотношение

$$
\ln \varphi(z)=-z^{2} \sigma^{2} / 2+O(1 / \hat{\Lambda})
$$

(выполняющееся равномерно в любом конечном интервале $|z| \leqslant R$ ) означает, что семиинварианты $\varkappa_{r}(T), r \geqslant 3$, величины

$$
T=\left(\zeta^{\prime}-\hat{\Lambda}\right) \hat{\Lambda}^{-1 / 2}
$$

сходятся к нулю и при этом $\mathbf{E} T \rightarrow 0, \mathbf{D} T \rightarrow \sigma^{2}$. Поэтому, согласно теореме 1 работы [7], моменты величины $T$ сходятся к моментам нормального распределения. 
Доказательство второй и третьей части теоремы 6 аналогично доказательству ее первой части. При этом в случае $a=0$ используется равенство (94).

Доказательство теоремы 7 вытекает из леммы 10 и третьей части теоремы 1.

Доказательство теоремы 8. Пользуясь теоремой 3 , леммой 13 и третьей частью теоремы 6 , получаем, что

$$
\begin{aligned}
\mathbf{P}\left\{z_{1}<(\eta-\Lambda) / \sqrt{\Lambda}<z_{2}\right\} & =\Lambda^{-1} \sum_{\substack{\Lambda+z_{1} \sqrt{\Lambda}<k<\Lambda+z_{2} \sqrt{\Lambda}\\
}} \mathbf{P}\{\xi=k\}(1+o(1)) \\
& =\sum_{\substack{\Lambda+z_{1}<k<\Lambda+z_{2} \sqrt{\Lambda}\\
}} \mathbf{P}\{\xi=k\}(1=o(1))
\end{aligned}
$$

где $\Phi(z)$ - функщия стандартного нормального распределения. Отсюда следует (13).

Доказательство теоремы 9 вытекает из равенства (105) и теоремы 8.

Автор признателен В. Г. Михайлову за ряд важных замечаний.

\section{Список литературы}

1. Копытцев В. А., О числе решений системы линейных булевых уравнений в множестве векторов, обладающих заданным числом единиц. Дискретная математика (2002) 14, №4, 87-109.

2. Балакин $\Gamma$. В., Введение в теорию случайных систем уравнений. Труды по дискретной математике (1997) 1, 1-18.

3. Севастьянов Б. А., Предельный закон Пуассона в схеме сумм зависимых случайных величин. Теория вероятностей и ее применения (1972) 17, №4, 733-738.

4. Колчин В. Ф., Севастьянов Б. А., Чистяков В. П., Случайные размещения. Москва, Наука, 1976.

5. Михайлов В. Г., Явные оценки в предельных теоремах для сумм случайных индикаторов. Обозрение прикладной и промышленной математики (1994) 1, №4, 580-617.

6. Сачков В. Н., Вероятностные методы в комбинаторном анализе. Москва, Наука, 1978.

7. Janson S., Normal convergence by higher semiinvariants with applications to sums of dependent random variables and random graphs. Ann. Probab. (1988) 16, №1, 305-312.

Статья поступила 30.12.2004. 